\title{
PATTERNS THEORY AND GEODESIC AUTOMATIC STRUCTURE FOR A CLASS OF GROUPS
}

\author{
MURRAY J. ELDER
}

\begin{abstract}
We introduce a theory of patterns in order to study geodesics in a certain class of group presentations. Using patterns we show that there does not exist a geodesic automatic structure for certain group presentations, and that certain group presentations are almost convex.
\end{abstract}

\section{INTRODUCTION}

In this article we examine the possibility of automatic structures in two intriguing examples. Wise's example is non-Hopfian and CAT(0) [11, so proving this group is not automatic would answer the open question: Is every CAT(0) group automatic? Conversely proving this group is automatic would answer another open question: Is every automatic group Hopfian (or residually finite)? Brady and Bridson's example is not biautomatic, not $\mathrm{CAT}(0)$, and has a quadratic isoperimetric function [1]. Finding an automatic structure for this group would answer a third open question of fundamental interest to automatic group theorists: Does every automatic group admit some biautomatic structure?

After some preliminary definitions we examine in some detail Brady and Bridson's example with a chosen generating set, which we denote by the pair $\left(G_{1,1}, X\right)$. We define the notion of a pattern in the Cayley graph and this idea enables us to prove that for the chosen generating set the full language of geodesics is not regular (Theorem 8.1), and further that for the chosen generating set the group can have no geodesic automatic structure (Corollary 8.3). We characterize the set of all patterns for $\left(G_{1,1}, X\right)$ (Theorem 9.1), and use this to prove that the pair is almost convex (Theorem 11.4). We remark that all of the above results can be replicated for Wise's example.

While these results go nowhere close to resolving the question of automaticity for these two examples, they provide much insight into the geodesic structure of this class of groups.

The author wishes to thank his advisor Walter Neumann for his encouragement and ideas throughout this project.

\section{Preliminaries: Automatic Groups}

Let $(G, X)$ be a group with finite generating set $X$, assume that $X$ is inverse closed, and let $X^{*}$ denote the set of all possible words in the letters of $X$, including the empty word. Let $\Gamma(G, X)$ be the Cayley graph for the pair $(G, X)$, metrized

Date: Published in International Journal of Algebra and Computation Vol 13, No 2 (2003) $203-230$.

2000 Mathematics Subject Classification. $20 \mathrm{~F} 65$.

Key words and phrases. Automatic group, regular language of geodesics, almost convex. 
by giving each edge unit length and endowing it with the path metric. That is, the distance between any two points is the infimum of the lengths of paths between these points in the graph. A word in $X^{*}$ describes a path in the Cayley graph and vice versa. Paths can be parameterized by non-negative $t \in \mathbb{R}$ by defining $w(t)$ as the point distance $t$ along the path $w$ from the identity if $t<|w|$ and $w(t)=\bar{w}$ if $t \geq|w|$, where $\bar{w}$ is the endpoint of $w$ and $|w|$ is the length of the path $w$ which is equal to the number of letters in the word $w$. If two words $w, u$ evaluate to the same group element in $G$ then we write $w={ }_{G} u$.

Paths $w$ and $u$ are said to $k$-fellow travel if $d(w(t), u(t)) \leq k$ for each $t \in \mathbb{R}$ with $t \geq 0$. The two paths are asynchronous $k$-fellow travelers if there is a non-decreasing proper continuous function $\phi:[0, \infty) \rightarrow[0, \infty)$ such that $d(w(t), u(\phi(t))) \leq k$. This means that any point on $w$ is within $k$ of some point on $u$ and vice versa. We imagine the two paths traveling at different speeds (but not backtracking) to keep within $k$ of each other.

Definition 2.1 (Fellow traveler property). A language $L \subset X^{*}$ enjoys the (asynchronous) fellow traveler property if there is a constant $k$ such that for each $w, u \in L$ with $d(\bar{w}, \bar{u}) \leq 1$ in $\Gamma(G, X), w$ and $u$ (asynchronously) $k$-fellow travel.

A language $L \subset X^{*}$ on any finite alphabet $X$ is said to be regular if it is the set of words accepted by some finite state automaton. See [6] for details.

A simple yet powerful result about regular languages is the following.

Lemma 2.2 (Pumping Lemma). Let $M$ be a finite state automaton on an alphabet $X$, having $n$ states. If $w \in L(M)$ is a word of length greater than $n$ then we can write $w=u z v$ with $|z|>0$ and $u z^{i} v \in L(M) \forall i \geq 0$.

Proof. If $|w|>n$ then as $M$ reads $w$ it must pass through the same state more than once. Let $u$ be the prefix of $w$ until it reaches this state. Let $z$ be the next part of $w$ until it gets back to the repeated state. Then $v$ is the remaining part of $w$. Now since $w=u z v$ ends in an accept state so does $u z^{i} v$ for all $i \geq 0$.

The set of regular languages is known as a formal language. In general the set of words on an alphabet that are accepted by some kind of computing machine is a formal language. Formal language classes can be arranged in a hierarchy known as the "Chomsky hierarchy", in order of increasing complexity. The complexity of a language can be seen in terms of the complexity of the machine which accepts it. Since finite state automaton are the simplest type of computing machines the class of regular languages is at the start of the Chomsky hierarchy. More details can be found in 8 .

Definition 2.3 (Automatic). Let $(G, X)$ be a group with finite generating set $X$, and let $L \subset X^{*}$ be some set of words on $X^{*}$ such that

(1) $L$ is regular

(2) $L$ has the (asynchronous) fellow traveler property

(3) $L$ surjects to $G$.

Then $L$ is an (asynchronous) automatic structure for $(G, X)$.

If $G$ has an automatic structure with respect to one generating set then it has an automatic structure with respect to every generating set [6] so we say that $G$ is (asynchronously) automatic if it has an (asynchronous) automatic structure for some generating set. 
Definition 2.4 (Biautomatic). $L \subset X^{*}$ is a biautomatic structure for $(G, X)$ if $L$ and $L^{-1}$ are both automatic structures for $(G, X)$.

If $L$ is an automatic structure then the only thing to check for biautomaticity is that $L^{-1}$ has the fellow traveler property.

If $G$ is automatic then it has at most a quadratic isoperimetric function [6].

If $L \subset X^{*}$ is a language surjecting to $G$ and has the fellow traveler property then we say $L$ is a combing for $G$. If $L^{-1}$ also has the fellow traveler property then we say $L$ is a bicombing.

A group is $C A T(0)$ if it acts properly discontinuously co-compactly by isometries on some $\operatorname{CAT}(0)$ metric space (See [2]). If $G$ is $\operatorname{CAT}(0)$ then space on which is acts admits a bicombing by geodesics. This does not prove that CAT(0) groups are biautomatic or even automatic since the geodesic bicombing may not project onto the 1-skeleton and also be a regular language. Therefore whether $\operatorname{CAT}(0)$ groups are (bi)automatic is presently an open problem.

Example 1. Let $\left(G_{1,1}, X\right)$ be the group with presentation

$$
\left\langle a, b, c, d, s, t \mid c=a b=b a, d=a b^{-1}, s^{-1} a s=c, t^{-1} a t=d\right\rangle .
$$

$G_{1,1}$ has no biautomatic structure, has a quadratic isoperimetric function [1], and is not $\mathrm{CAT}(0)$ [7. It is asynchronously automatic [4] and the proof of this is omitted.

Example 2. Let $\left(G_{W}, Y\right)$ be the group with presentation

$$
\left\langle a, b, c, d, s, t \mid c=a b=b a, d=c^{2}, s^{-1} a s=d, t^{-1} b t=d\right\rangle .
$$

$G_{W}$ is non-Hopfian, $\operatorname{CAT}(0)$ [1] and asynchronously automatic [4].

\section{Preliminaries: HNN extensions}

Definition 3.1 (Multiple HNN extension). Let $(A, Z)$ be a group with finite generating set $Z$ and relations $R$, let $U_{1}, \ldots, U_{n}, V_{1}, \ldots, V_{n}$ be subgroups of $A$ and let $\phi_{i}: U_{i} \rightarrow V_{i}$ be an isomorphism for each $i$. The group $(G, X)$ with presentation

$$
\left\langle Z, s_{1}, \ldots, s_{n} \mid R, s_{i}^{-1} u_{i} s_{i}=\phi_{i}\left(u_{i}\right) \forall u_{i} \in U_{i}, \forall i\right\rangle
$$

is a multiple $H N N$ extension of $(A, Z)$. The generators $s_{i}$ are called stable letters, and the pairs of $U_{i}, V_{i}$ are called associated subgroups.

If each $U_{i}$ is finitely generated by $\left\{u_{i_{j}}\right\}$ and $\phi_{i}\left(u_{i_{j}}\right)=v_{i_{j}}$ then $V_{i}$ is finitely generated by $\left\{v_{i_{j}}\right\}$. Thus $(G, X)$ has the finite presentation

$$
\left\langle Z, s_{1}, \ldots, s_{n} \mid R, s_{i}^{-1} u_{i_{j}} s_{i}=v_{i_{j}} \forall i, \forall j\right\rangle .
$$

Theorem 3.2 (Britton's Lemma). Let $(G, X)$ be a multiple HNN extension with the presentation in Definition 3.1 above. If $w \in X^{*}$ is freely reduced and $w={ }_{G} 1$ then $w$ contains a sub-word of the form $s_{i}^{-1} u_{i_{j}} s_{i}$ or $s_{i} v_{i_{j}} s_{i}^{-1}$ for some non-trivial $u_{i_{j}} \in U_{i}$ or $v_{i_{j}} \in V_{i}$.

A proof can be found in [9]. We call the sub-word $s_{i}^{-1} u_{i_{j}} s_{i}$ or $s_{i} v_{i_{j}} s_{i}^{-1}$ a pinch.

Definition 3.3. A word that does not admit any pinches is called stable letter reduced. If two words have the same sequence of stable letters then we say they have parallel stable letter structure. 
Example 3. If $n=1$ and $A=\mathbb{Z}$ then $(G, X)$ is a Baumslag-Solitar group. If $A=\mathbb{Z}^{r}$ for some $r>1$ and $n>1$ then $(G, X)$ is sometimes called a generalized Baumslag-Solitar group.

Example 4. $G_{1,1}$ is a double HNN extension of $\mathbb{Z}^{2} \cong\langle a, b, c, d| c=a b=b a, d=$ $\left.a b^{-1}\right\rangle$ associating cyclic subgroups $\langle a\rangle,\langle c\rangle$ and $\langle a\rangle,\langle d\rangle$.

Example 5. $G_{W}$ is a double HNN extension of $\mathbb{Z}^{2} \cong\left\langle a, b, c, d \mid c=a b=b a, d=c^{2}\right\rangle$ associating cyclic subgroups $\langle a\rangle,\langle d\rangle$ and $\langle b\rangle,\langle d\rangle$.

The Cayley graph of a multiple HNN extension can be viewed in the following way. For each presentation of a group we can construct the "presentation 2-complex", where the 1-skeleton of its universal cover is the Cayley graph, and the 2 -skeleton is sometimes called the "Cayley complex" or the "filled Cayley graph". If $(G, X)$ is a multiple $\mathrm{HNN}$ extension of $(A, Z)$, the presentation 2-complex is formed by taking the presentation 2-complex for $(A, Z)$ and attaching $n$ annuli by appropriate edge gluings. In the universal cover we see copies of the "base space"

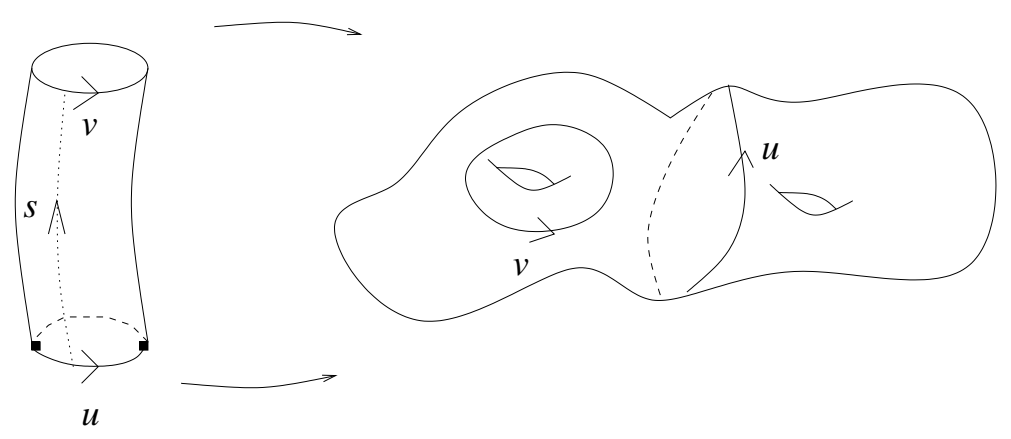

FiguRe 1. Presentation 2-complex for a multiple HNN extension

or the 2-complex for $(A, Z)$ glued together by copies of the annuli, which attach themselves along associated subgroups of $(A, Z)$. For example, the presentation 2-complex for $\left(G_{W}, Y\right)$ can be seen as two annuli (or cylinders) with boundaries labeled $a, d$ and $b, d$ respectively, attached to a torus with a triangle $c^{2} d^{-1}$ glued to it, by identifying the loops $a, b$ and $d$, as in Figure 2. The universal cover of the torus with a triangle attached is a plane with "bumps" on both sides, and the universal cover of each cylinder gives a bi-infinite "strip". So we can view any such multiple HNN extension as being made up of base-group planes glued together along associated subgroups by stable letter strips.

Definition 3.4 (Strip equidistant). Let $(G, X)$ be a multiple HNN extension with the presentation in Definition 3.1 above. If $\left|u_{i}\right|=\left|\phi\left(u_{i}\right)\right|$ for all $i$ then we say $(G, X)$ has a strip equidistant presentation.

Note that if $(G, X)$ has a strip equidistant presentation then if a word $w \in X^{*}$ admits a pinch then it can be shortened by 2 , so geodesics are stable letter reduced.

Definition 3.5 (Geodesic subgroup). Let $(G, X)$ be any group with generating set $X$. A subgroup $A$ of $G$ with generating set $Y \subseteq X^{*}$ is geodesic in $(G, X)$ if each element of $A$ has a geodesic word $w \in Y^{*}$ evaluating to it. 


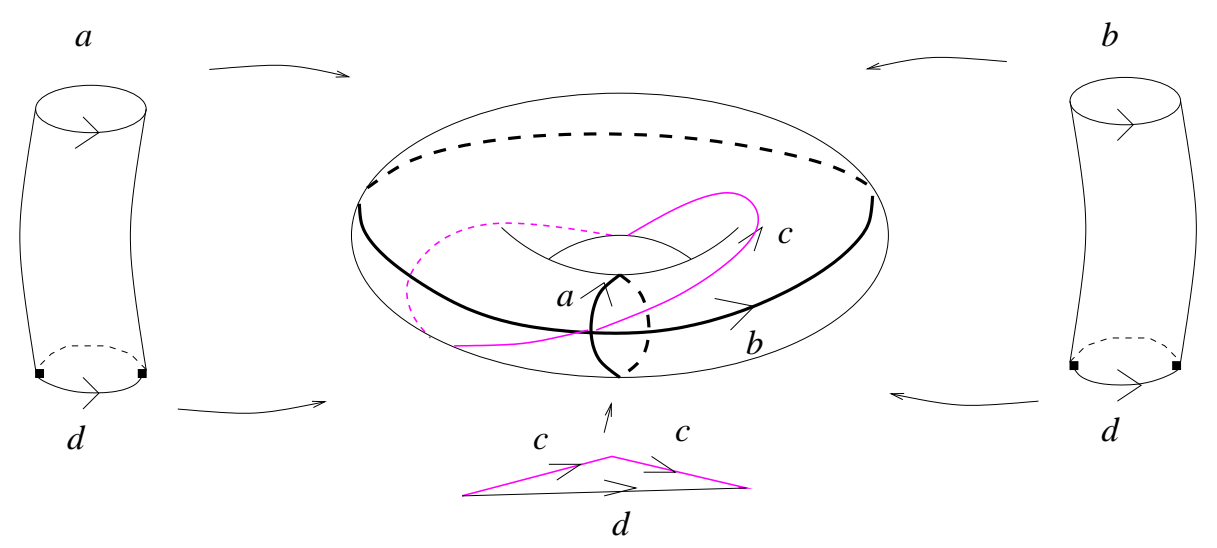

Figure 2. Presentation 2-complex for $\left(G_{W}, Y\right)$

Definition 3.6 (Totally geodesic). Let $(G, X)$ be any group with generating set $X$. A subgroup $A$ of $G$ with generating set $Y \subseteq X^{*}$ is totally geodesic in $(G, X)$ if every geodesic word $w \in X^{*}$ evaluating to an element of $A$ is an element of $Y^{*}$. A subspace of the Cayley graph which is a copy of $\Gamma(A, Y)$ inside $\Gamma(G, X)$ is called totally geodesic if $(A, Y)$ is.

It is clear that totally geodesic subgroups are geodesic.

Example 6. $\left(G_{1,1}, X\right)$ has a strip equidistant presentation, and the subgroup $\mathbb{Z}^{2} \cong$ $\left\langle a, b, c, d \mid a b=b a, c=a b, d=a b^{-1}\right\rangle$ is totally geodesic. The associated subgroup $\langle a\rangle$ of $\mathbb{Z}^{2}$ is not totally geodesic in $\left(\mathbb{Z}^{2},\{a, b, c, d\}\right)$, since $c^{i} d^{i}={ }_{Z^{2}} a^{2 i}$. The subgroups $\langle a\rangle,\langle c\rangle,\langle d\rangle$ are geodesic in $\left(\mathbb{Z}^{2},\{a, b, c, d\}\right)$.

Example 7. $\left(G_{W}, Y\right)$ has a strip equidistant presentation, the subgroup $\mathbb{Z}^{2} \cong$ $\left\langle a, b, c, d \mid a b=b a, c=a b, d=c^{2}\right\rangle$ is totally geodesic in it, and all associated cyclic subgroups of $\mathbb{Z}^{2}$ are totally geodesic in $\left(\mathbb{Z}^{2},\{a, b, c, d\}\right)$.

\section{Geodesics And Sequences}

Throughout this section $(G, X)$ will be a multiple HNN extension of $\left(\mathbb{Z}^{n}, Y\right)$ with a strip equidistant presentation, where $Y$ is some finite generating set for $\mathbb{Z}^{n}$. Let a $\mathbb{Z}^{n}$-plane refer to the copies of $\Gamma\left(\mathbb{Z}^{n}, Y\right)$ in $\Gamma(G, X)$.

Lemma 4.1. $\mathbb{Z}^{n}$-planes are totally geodesic in $\Gamma(G, X)$.

Proof. Suppose $w$ is a geodesic from $p$ to $q$ in a $\mathbb{Z}^{n}$-plane, and $w$ does not lie in the plane. Then $w$ must contain a stable letter. Let $u$ be a path in the $\mathbb{Z}^{n}$-plane from $p$ to $q$. Then $w u^{-1}={ }_{G} 1$ so by Britton's Lemma it contains a pinch. Since $u$ has no stable letters then $w$ must contain the pinch. But since the presentation is strip equidistant performing a pinch would shorten $w$ by 2 , which is a contradiction.

Corollary 4.2. A geodesic path in $\Gamma(G, X)$ visits no $\mathbb{Z}^{n}$-plane twice.

Proof. If a path leaves some $\mathbb{Z}^{n}$-plane at a point $p$ then re-enters at a point $q$, then it cannot be geodesic by the Lemma. 
Lemma 4.3. Let $g \in G$. Every stable letter reduced path from 1 to $g$ crosses the same succession of planes and strips.

Proof. We proceed by induction on the number of stable letters in a stable letter reduced word. If $w$ is a stable letter reduced and has no stable letters, and if $u$ is a stable letter reduced word for $w$, then $w u^{-1}={ }_{G} 1$ so if $w u^{-1}$ contains any stable letters it must contain a pinch. Thus $u$ has no stable letters so both paths stay in the base $\mathbb{Z}^{n}$-plane. Now assume the hypothesis for stable letter reduced words having at most $k-1$ stable letters. Suppose $w$ is stable letter reduced and has $k$ stable letters, and $u$ is a stable letter reduced word for $w$. Write $w=w_{0} r_{1} \ldots w_{n-1} r_{n} w_{n}$ and $u=u_{0} t_{1} \ldots u_{m-1} t_{m} u_{m}$. Now $w u^{-1}=_{G} 1$ and contains stable letters so there is a pinch, so choose $i, j$ maximal such that $r_{i}\left(w_{i} \ldots r_{n} w_{n} u_{m}^{-1} t_{m}^{-1} \ldots u_{j}^{-1}\right) t_{j}^{-1}$ is a pinch. Then $\left(w_{i} \ldots r_{n} w_{n} u_{m}^{-1} t_{m}^{-1} \ldots u_{j}^{-1}\right)$ is a subgroup element so if it contains a stable letter it must contain a pinch, so $i, j$ were not maximal.

Thus $r_{n} w_{n} u_{m}^{-1} t_{m}^{-1}$ is a pinch. Let $y=r_{n} w_{n} u_{m}^{-1} t_{m}^{-1}$ be the subgroup element which runs along the other side of the strip. So $w, u$ end in the same plane and both cross the same last strip. Now let $w=w^{\prime} r_{n} w_{n}, u=u^{\prime} t_{m} u_{m}$. Then $w^{\prime} y=u^{\prime}$ are stable letter reduced and $w^{\prime} y$ has $k-1$ stable letters so by hypothesis $u^{\prime}, w^{\prime} y$ and $w^{\prime}$ cross the same succession of strips and planes to get to the same last strip, so we are done.

It follows easily that all stable letter reduced paths to a point have parallel stable letter structure, but the result above is stronger.

Corollary 4.4. All geodesics for a group element $g \in G$ cross the same succession of strips and planes from 1 to $\mathrm{g}$.

Thus to keep track of all potentially shorter paths when reading a word one can restrict one's attention to a single "branch" of the Cayley graph.

Now lets suppose we are building some (finite) machine that can decide whether or not a given (stable letter reduced) word is geodesic. We start at 1 in the Cayley graph and read until we hit the first stable letter. This corresponds to crossing the first strip in the Cayley graph.

Definition 4.5 (Witness). We call another word $u$ a witness for $w$ if at some point $\bar{u}=\overline{w(t)}$ and $|u|<|w(t)|$.

We think of $u$ as witnessing the fact that $w$ is not a geodesic. By the corollary all potential witnesses for $w$ must cross this first strip also.

For example, consider the group $\left(G_{1,1}, X\right)$, and the word $w=c^{2} b s^{-1} w^{\prime}$ shown in Figure 3. We need to keep track of all the geodesics from 1 to any "crossing point" along this strip, since one of these could potentially be a witness. Now it doesn't matter which path one chooses to reach each crossing point; we only need to know the length of a geodesic from 1 to this point. In fact, we only need to know the relative difference between the length of of geodesic from 1 to this point, and the length of $w$ to its crossing point.

So to keep track of all potential witnesses, we simply record the relative distances from each crossing point along the strip back to 1. For example, in Figure 4 we have written these numbers at each crossing point on the strip.

We are almost ready to make our main definition, but before we do, recall that we are thinking about building a (finite) machine that could store this information and 


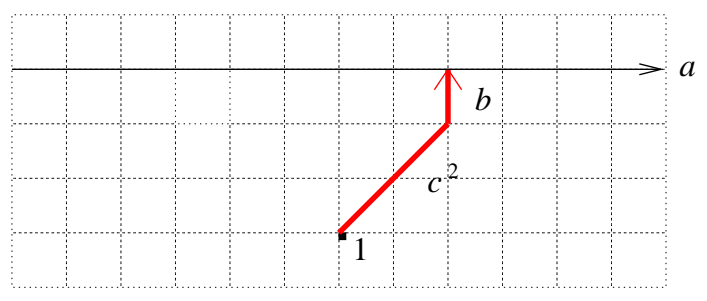

Figure 3. $w=c^{2} b s^{-1} w^{\prime}$ in $\left(G_{1,1}, X\right)$

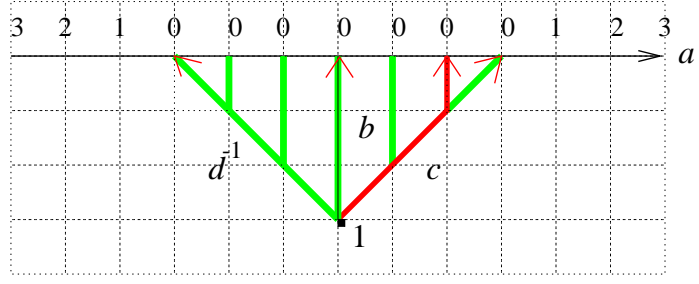

Figure 4. (All) geodesics to the first strip in $\left(G_{1,1}, X\right)$

so keep track of witnesses as it reads a word $w$. So instead of recording the actual distances as we have written them in Figure 4 we instead choose an orientation of the strip, then record the difference between adjacent numbers along the strip.

Definition 4.6 (Sequence). A sequence is a bi-infinite sequence of numbers $(0, \pm 1)$ which correspond to the difference between the relative distances from adjacent crossing points along a strip back to 1 in the Cayley graph.

For example, the sequence for the strip in Figure 4 is

$$
\ldots,-1,-1,0,0,0,0,0,0,1,1, \ldots
$$

We write this more neatly as $(-1)(0)^{6}(1)$, where the terminals are always understood to be infinite.

\section{Initial SEquences And PATterns for $\left(G_{1,1}, X\right)$ And $\left(G_{W}, Y\right)$}

The diversity of sequences that can arise in a generic multiple HNN extension depends on the presentation. For the group $\left(G_{1,1}, X\right)$ the first strip could be glued to either the subgroup $\langle a\rangle,\langle c\rangle$ or $\langle d\rangle$. Each case is shown in Figure 5 and we see that all sequences of the form $(-1)(0)^{k}(1)$ are possible for any $k=0,1,2 i ; i \in \mathbb{N}$. We say that these sequences all have (or "belong to") the same "pattern", and we will define the idea of a pattern presently. Remember that to build a (finite) machine which keeps record of certain (bi-infinite) sequences as it reads a word would need to be able to record arbitrarily large values of $k$. So instead of recording sequences we could try to record the patterns.

Definition 5.1 (Pattern). A pattern is an expression of the form $(-1)\left(p_{1}\right)\left(p_{2}\right) \ldots\left(p_{k}\right)(1)$ where the $p_{i}$ are finite words in $\{0, \pm 1\}$, the $\left(p_{i}\right)$ terms represent any finite number of repeats of $p_{i}$, and the terminals are infinite. A pattern occurs in a Cayley graph if there is some sequence realizing this pattern 


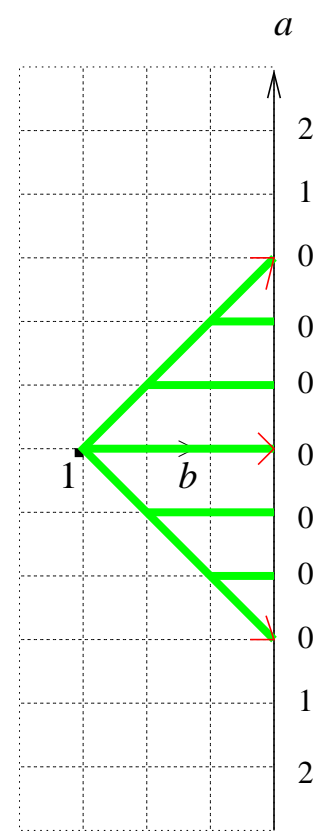

2

0

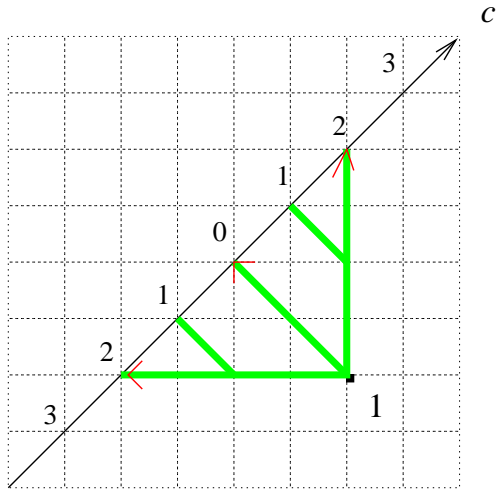

$d$

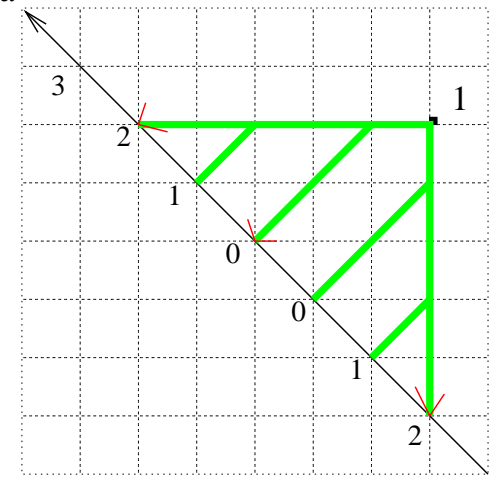

Figure 5. Initial sequences for $\left(G_{1,1}, X\right)$

with certain (positive) exponents on each internal term. The set of all patterns that can occur for a group presentation will be called its patterns theory.

For $\left(G_{1,1}, X\right)$ each initial sequence has the pattern $(-1)(0)(1)$, as noted above.

For the group $\left(G_{W}, Y\right)$ the first strip could be glued to either the subgroup $\langle a\rangle,\langle b\rangle$ or $\langle d\rangle$. Each case is shown in Figure 6 and we see that the possible initial sequences are $(-1)(0)^{k}(1)$ and $(-1)(10)^{k}(1)$ for any non-negative integer $k$. Thus $\left(G_{W}, Y\right)$ has two distinct initial patterns: $(-1)(0)(1)$ and $(-1)(10)(1)$.

So one can see that these two examples have different initial patterns. Next we will consider what happens on the next strip that a word crosses, and one might suspect that the new patterns that arise here will vary in each example. Therefore to avoid confusion we will stick with one example and examine the set of all possible sequences and patterns that can occur. 

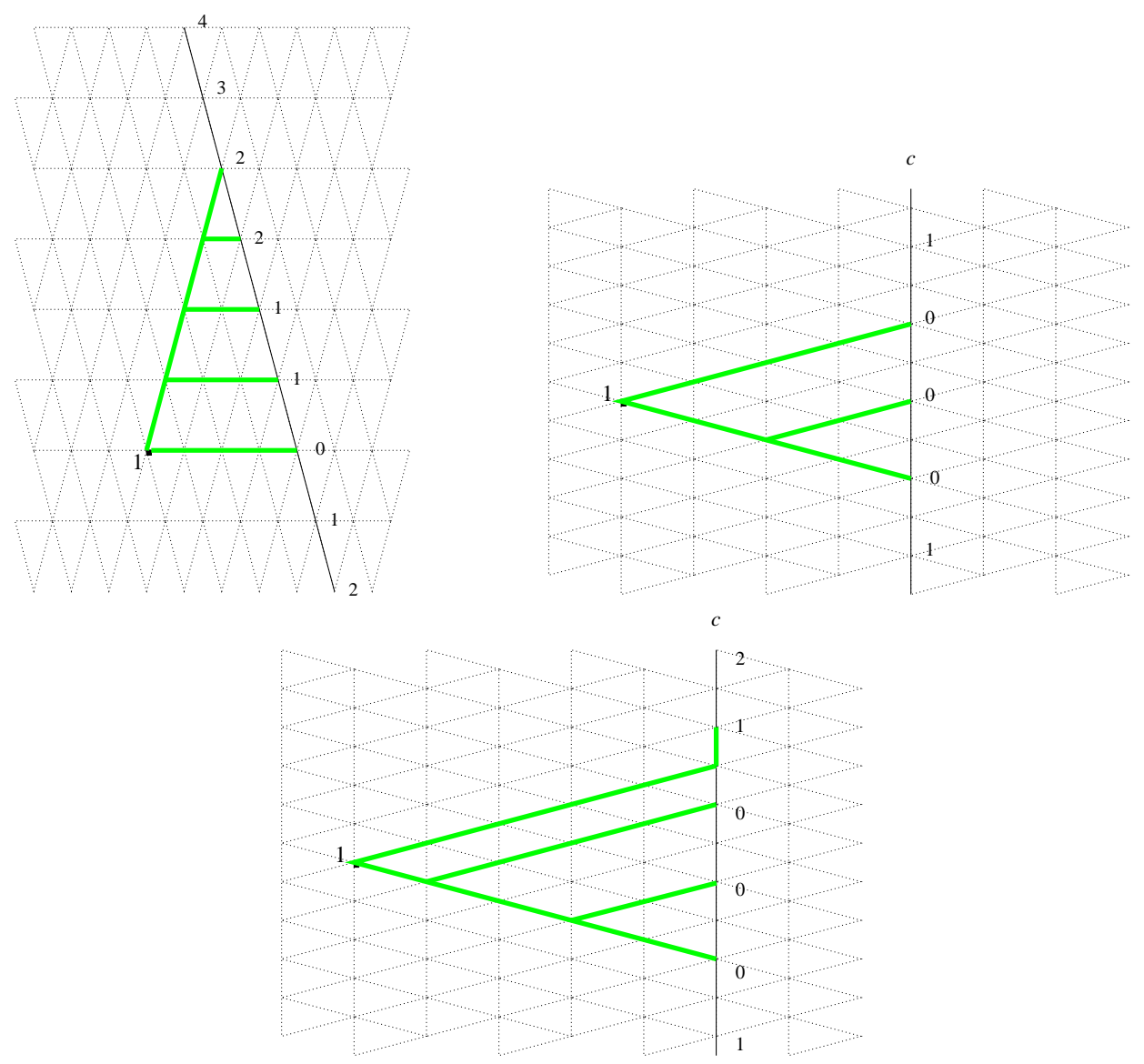

FIGURE 6 . Initial sequences for $\left(G_{W}, Y\right)$.

Also for clarity in the figures we write the actual relative distances back to 1 , and leave it to the reader to determine the sequence in each case.

\section{Moves}

We wish to characterize all sequences that occur in the Cayley graph of $\left(G_{1,1}, X\right)$. We will prove that only certain patterns occur, that is, all sequences are of a certain form.

All geodesics start in the plane containing 1 . The strip we use to exit this plane is called the initial strip. There are three types of strips to exit, and all have the same type of pattern, as seen in Figure 5 .

Let an $x$-line mean a bi-infinite straight path in the Cayley graph corresponding to the path $x^{i}$. The notation $x \rightarrow y$ means that we enter the plane via a strip glued to an $x$-line and exit the plane by a strip glued to a $y$-line.

Now there are only four ways to get from one plane to the next:

$1: a \rightarrow a, c \rightarrow c, d \rightarrow d$

$2: c \rightarrow a, d \rightarrow a$

$3: a \rightarrow c, a \rightarrow d$ 
$4: c \rightarrow d, d \rightarrow c$

We call these moves on the patterns, since they take an existing pattern and make a new one from it. We wish to write down all possible patterns that can be generated from the initial pattern by moves. Let's start with the initial pattern $(-1)(0)(1)$ and do some experimentation to see what kind of patterns are possible. Type 1 moves can be referred to as parallel moves since they occur when two concurrent strips are parallel on a plane. These are shown in Figure 7, In each case
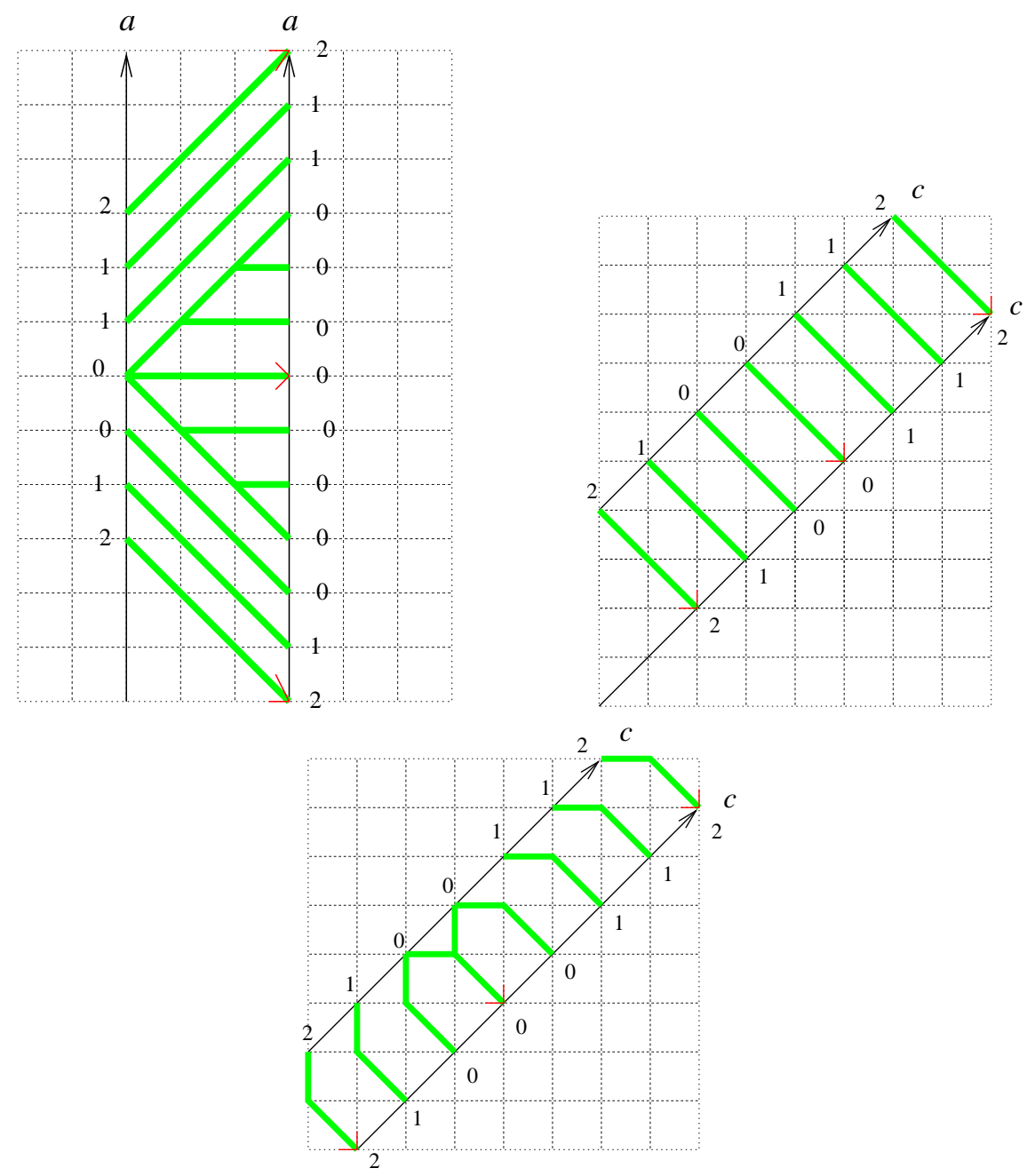

FiguRE 7. "Parallel moves"

we have just "expanded" the (0) sub-word of the initial pattern. So in this instance Move 1 acts as an identity move since it doesn't introduce any new structure. This may not be true when we have a more intricate pattern on the enter strip.

The type 2 move $c / d \rightarrow a$ gives a new pattern as shown in Figure 8 Applying Move 1 again just expands the (0) so preserves it. Applying Move 2 again to this gives Figure 9 . Applying Move 2 again gives Figure 10, Note that the power 


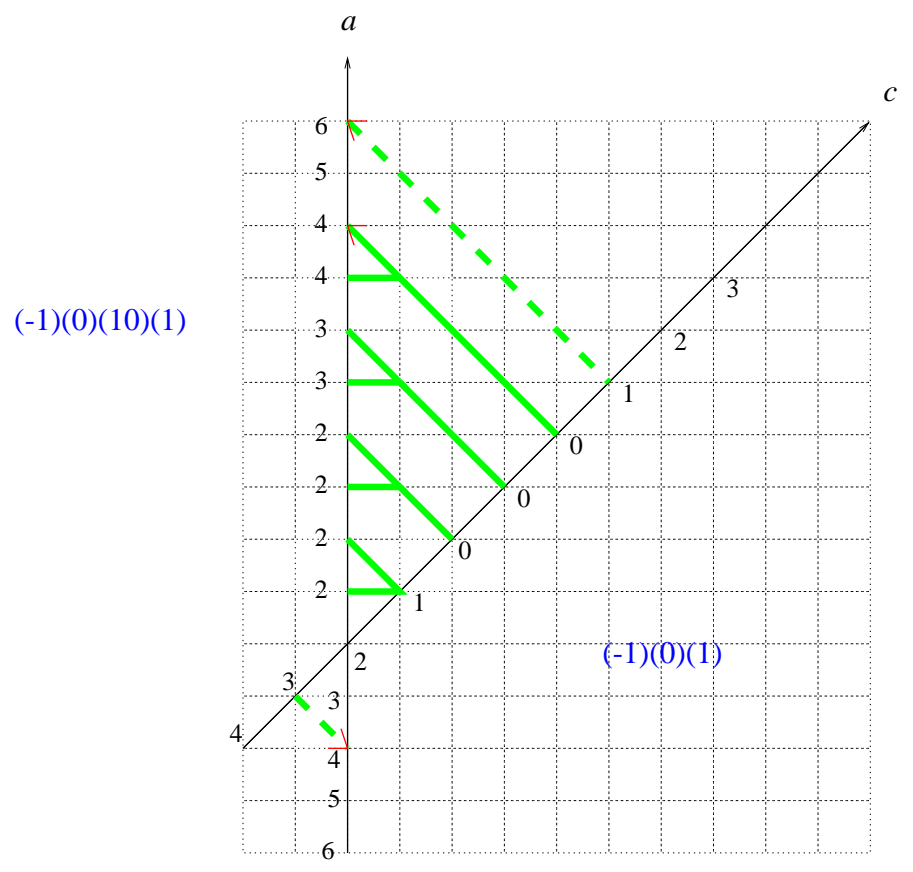

FiguRE 8. Move 2 gives $(-1)(0)(10)(1)$

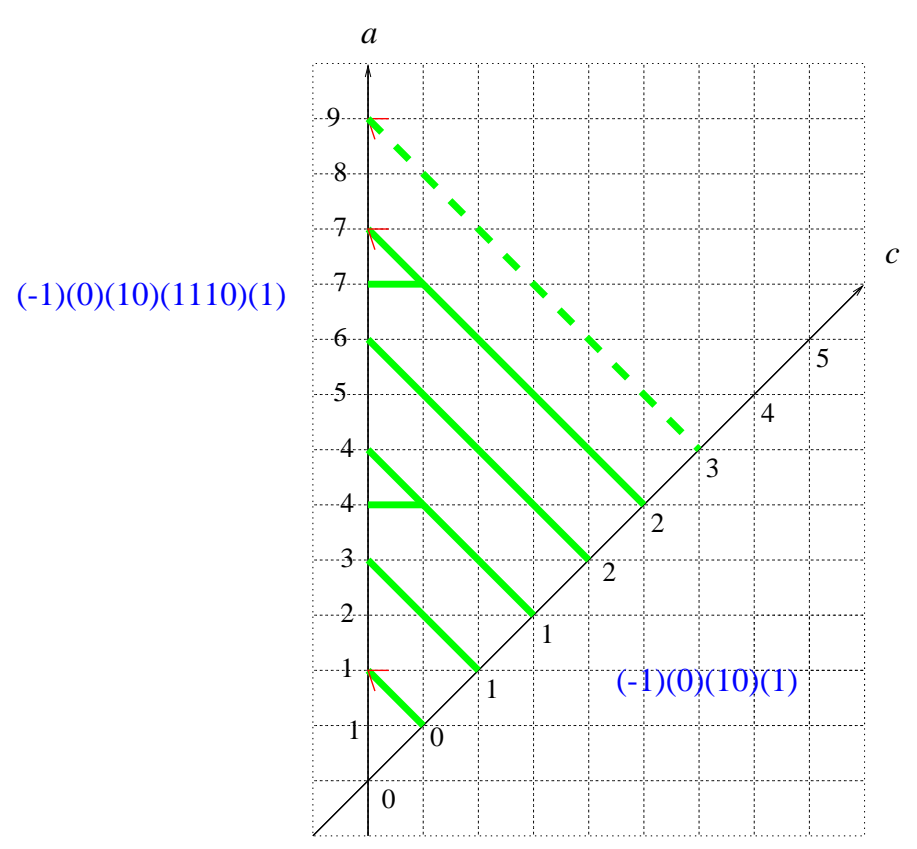

FiguRe 9. Another move 2 gives $(-1)(0)(10)(1110)(1)$ 
of seven in the pattern $\left(1^{7} 0\right)$ is not arbitrary in this case.

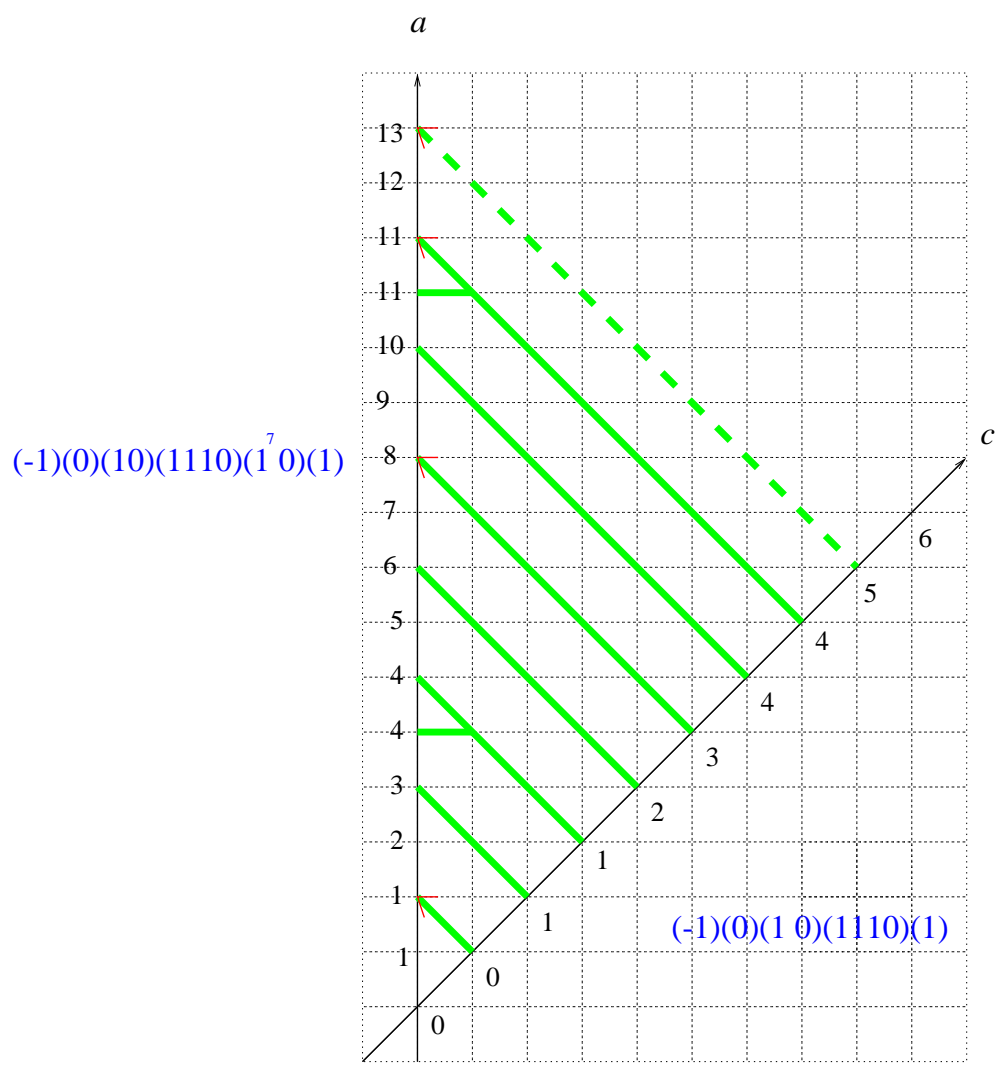

FIGURE 10. Another move 2 gives $(-1)(0)(10)(1110)\left(1^{7} 0\right)(1)$

Each iteration gives a new term between the two most recently introduced terms, that is, some kind of "rewrite" of the most recently introduced term. The reader is encouraged to keep going at this point, and see what weird and wonderful patterns can be generated. What happens when you apply $a \rightarrow c / d, c / d \rightarrow d / c$ and the parallel moves? How many jumps of 1 before a 0 are possible?

We will now introduce a further abstraction of sequences and patterns. The pattern

$$
(-1)(0)(10)(1110)\left(1^{7} 0\right)(1)
$$

above can be said to belong to the set of patterns of the form

$$
(-1)(0)(1,0)(1)
$$

where $(1,0)$ means any mixture of 0 's and 1's. This notation contains less information about the pattern's structure, but will be useful below. Note that applying a move 3 of the form $a \rightarrow c$ to this abbreviated pattern potentially can give $(-1)(0)(1,0,-1)(1)$ as shown in Figure 11, but in the old notation this would not occur. 
$\left(\begin{array}{llll}1 & 1 & 0 & 0\end{array}\right)$

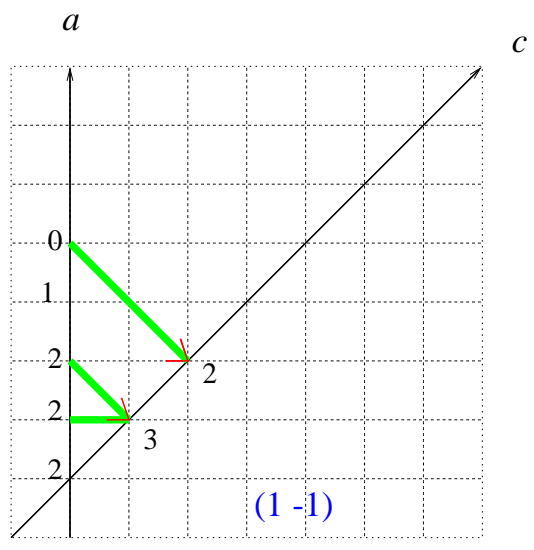

FiguRE 11. A potentially "bad" pattern

\section{Moves AS ReWriting RULes.}

We start with a conjecture about what patterns are allowed. Using this we can describe the moves more effectively, in terms of rewrite rules.

Conjecture 7.1. All patterns for $\left(G_{1,1}, X\right)$ are of the form

$$
(-1)(0,-1)(0)(1,0)(1) \text {. }
$$

Now assume the pattern $P=(-1) w_{1}(0) w_{2}(1)$ is of the conjectured form, so $w_{1}, w_{2}$ are words in $\{-1,0\},\{0,1\}$ respectively. Define $T=(-1)(0)(1)$ to be the trivial pattern, which is the pattern on the first strip for any geodesic.

Move 1: Parallel moves.

Each of these just increases the number of repeats of (0), so the pattern is preserved. Define Move 1 to be the identity move on the set of patterns, which corresponds to traversing parallel strips. So Move 1 "rewrites" any pattern $P=(-1) w_{1}(0) w_{2}(1)$ as itself, since our notation means there can be any finite number of zeroes in the middle parentheses.

We define a sub-pattern of $P=(-1) w(1)$ to be a pattern of the form $(-1) w^{\prime}(1)$, and a sub-sequence of $K=(-1) w(1)$ to be a sequence of the form $(-1) w^{\prime}(1)$, where $w^{\prime}$ is a sub-word of $w$.

Move 2: $c / d \rightarrow a$

Let $P=(-1) w_{1}(0) w_{2} w_{3}(1)$ be any pattern of the conjectured form.

Rewrite $P$ using the following rules:

$$
\begin{aligned}
& w_{1}(0) w_{2}:\left\{\begin{aligned}
-1 & \rightarrow-1-1 \\
0 & \rightarrow 0-1 \\
1 & \rightarrow 00
\end{aligned}\right. \\
& w_{3}:\left\{\begin{array}{lll}
0 & \rightarrow & 10 \\
1 & \rightarrow & 11
\end{array}\right.
\end{aligned}
$$

The subdivision of $w_{2} w_{3}$ corresponds to the point at which the two strips intersect on the plane, as in Figure 13 . 


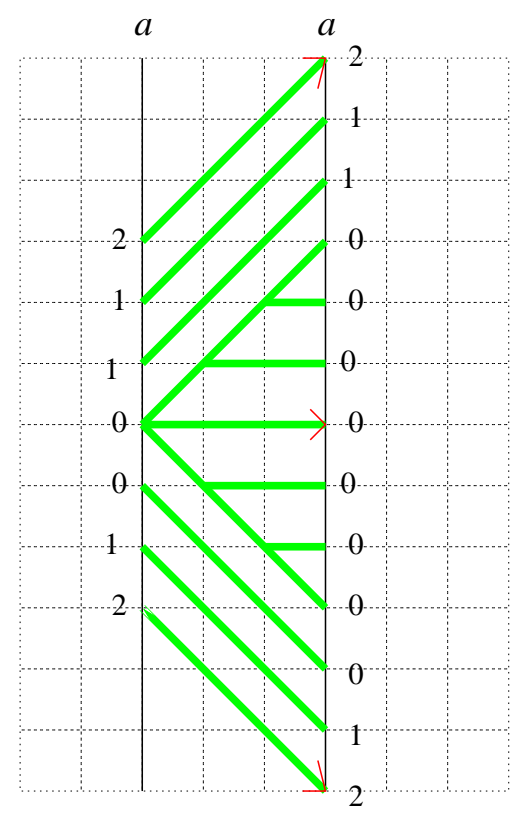

(a) $a \rightarrow a$

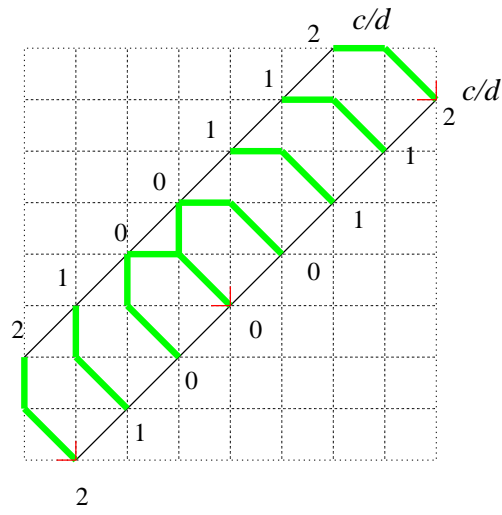

(b) $c / d \rightarrow c / d$

Figure 12. Move 1

Move 0: As defined a pattern has no orientation, so we define a Move 0 which rewrites the pattern in reverse. This move does not correspond to any strip crossing, it is merely a change of perspective. It is easily checked that moving 02 covers the remaining ways to go from $c / d \rightarrow a$.

Move 3. : $a \rightarrow c / d$

Take a sub-word (0) $w_{1}$ of a pattern in conjectured form. Rewrite:

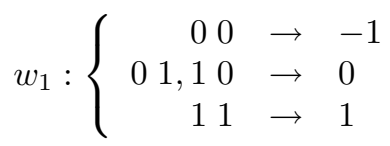

The subword $w_{1}$ in this case corresponds to the part of the pattern from (0) to the point $\mathrm{t}$ which the two strips intersect, as in Figure 14. Anything "below" this does not contribute to the new pattern.

Notice that this move could potentially give a pattern not in the conjectured form. For instance if we had a sub-pattern 1100 which is of conjectured form we have seen above this gives $1-1$ which is not of conjectured form .

Move 4: $c / d \rightarrow d / c$

Here we see that regardless of the previous pattern we always get a pattern $(-1)(1)$ which we can assume is the trivial pattern. Therefore only moves 2, 3 (and 0) can give non-trivial patterns.

So we now have a way to find every possible pattern for $\left(G_{1,1}, X\right)$; start with the initial (trivial) pattern and perform any number of moves $0,2,3$ in all possible ways, until we obtain a pattern that is not in conjectured form. It turns out that all patterns are in conjectured form, and we will prove this in section 9 . 


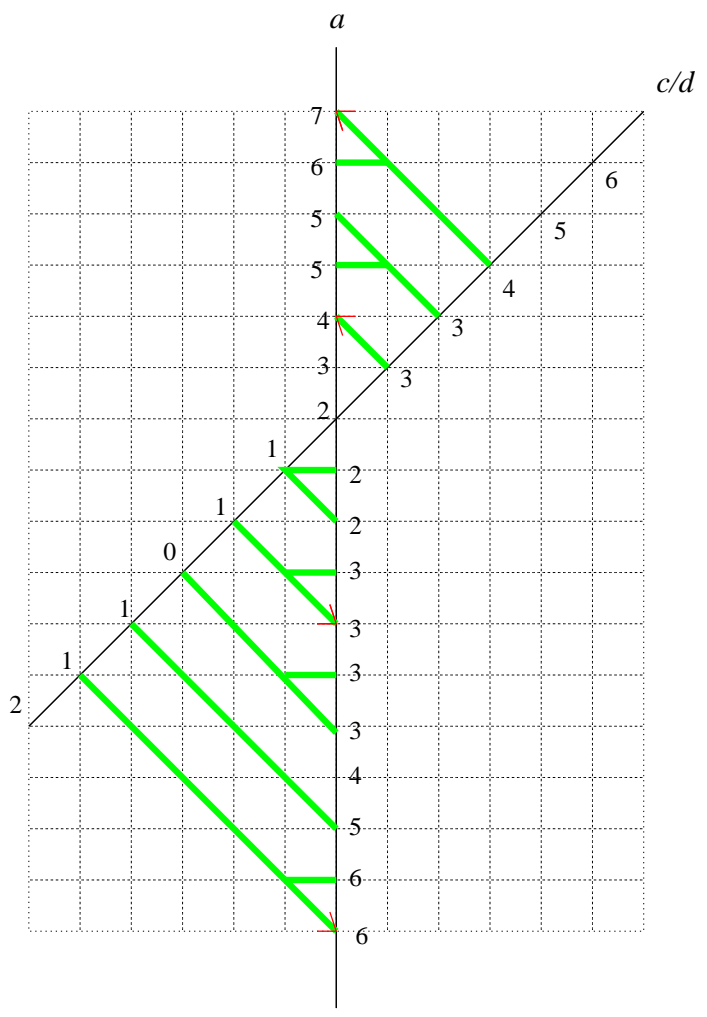

Figure 13. Move 2: $c / d \rightarrow a$

\section{Geodesic automatic structures}

Recall that the motivation for this approach was to show that some geodesic language for $\left(G_{1,1}, X\right)$ can be recognized by a finite machine.

Theorem 8.1. The full language of geodesics for $\left(G_{1,1}, X\right)$ is not regular.

Proof. We can find a sequence of the form $(-1)\left(1^{\left(2^{n}-1\right)} 0\right)(0)$ for arbitrarily large values of $n$, by performing some number of move 2 on the trivial sequence. We show the first three iterations of this in Figure 16. The three segments shown here are concatenated together in the Cayley graph.

This means there is a word $g=b^{-1} s^{n}$ which is shown in bold in Figure 16 , Now $g c^{\left(2^{n}-1\right)}$ is geodesic by the labeling of the sequence, and $g c^{2^{n}}$ is not. Then by the Pumping Lemma the language containing this (geodesic) word cannot be regular.

Theorem 8.2. For any $k>0$ there exist $g, g^{\prime} \in G_{1,1}$ such that $w \in X^{*}$ is the unique geodesic for $g$ and $w^{\prime} \in X^{*}$ is the unique geodesic for $g^{\prime}, d\left(g, g^{\prime}\right)=1$ and $w, w^{\prime}$ do not $k$-fellow travel.

Corollary 8.3. There is no geodesic automatic structure for $\left(G_{1,1}, X\right)$.

Proof. We continue with moves on the sequence used in the preceding proof. Intuitively we can now "undo" the $1^{k} 0$ sequence by Move 3 's to get two geodesics which 


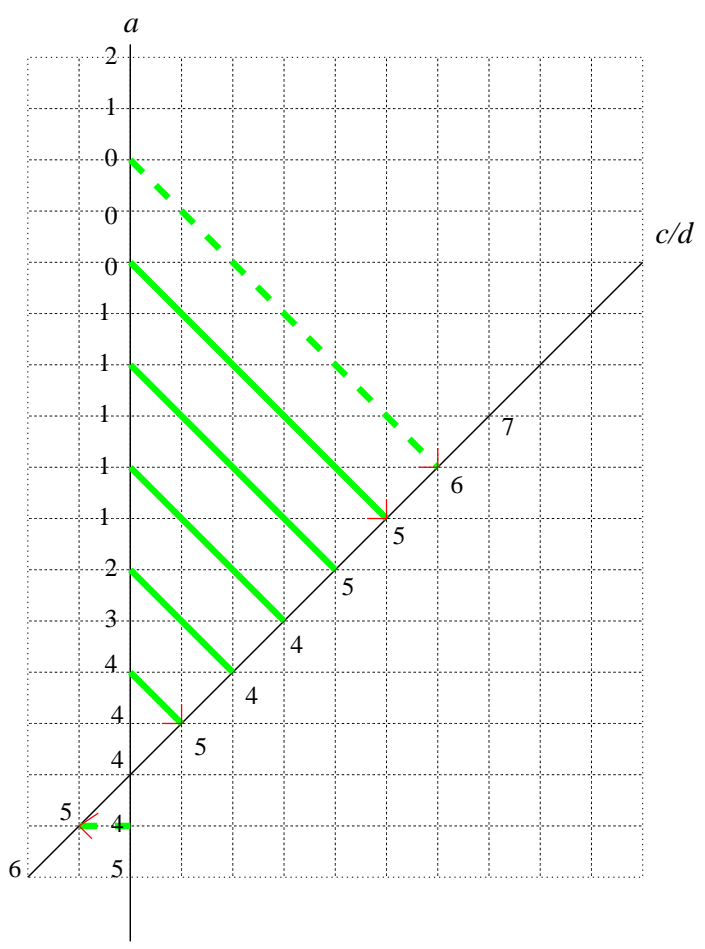

Figure 14. Move 3: $a \rightarrow c / d$

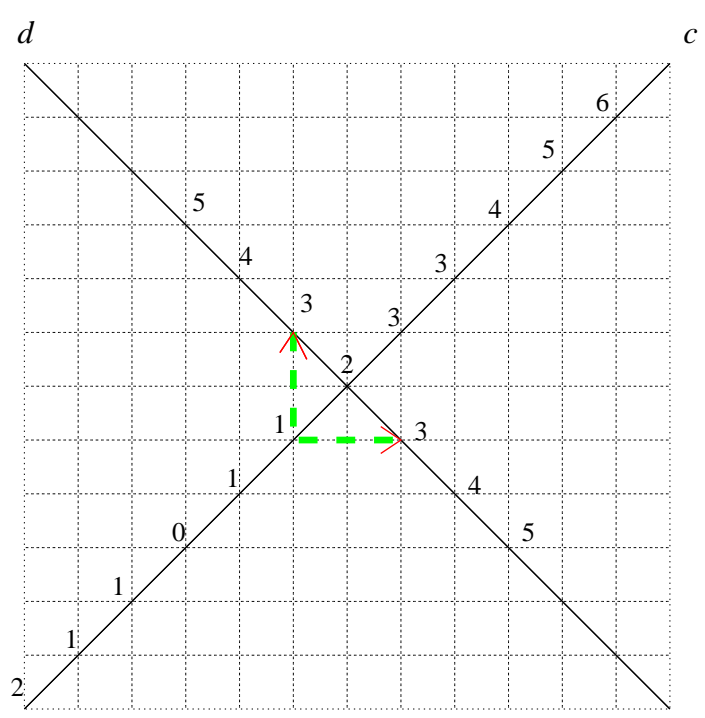

Figure 15. Move 4: $c / d \rightarrow d / c$

are the unique geodesics from 1 to their endpoints, ending an edge apart that don't fellow travel. 

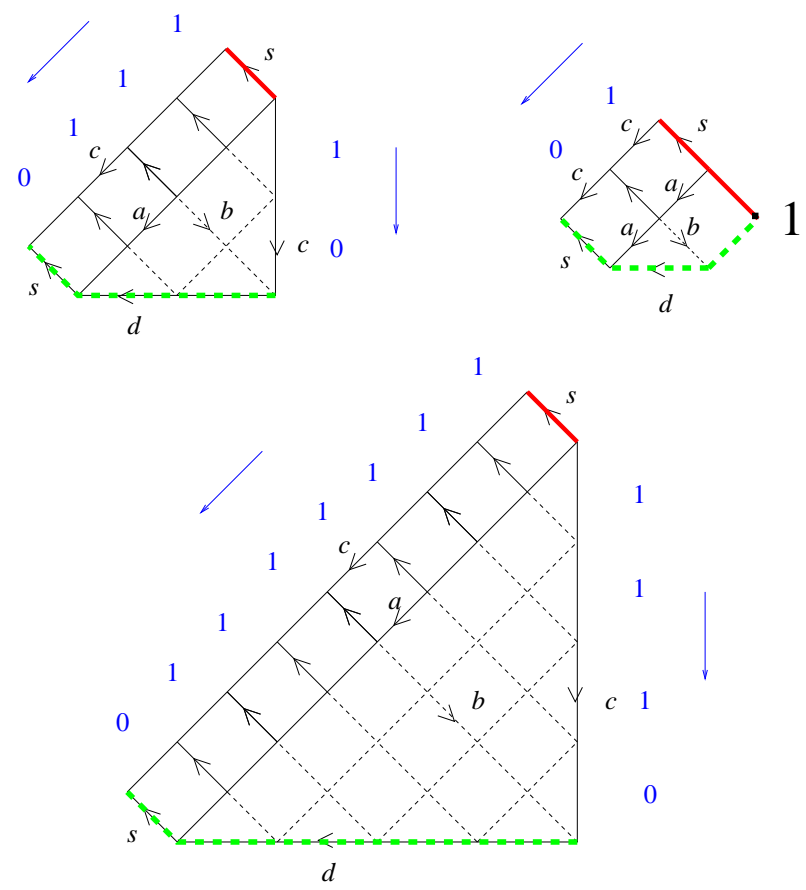

FiguRE 16. Finding a sequence $1^{k} 0$

The previous argument gives us two paths to a strip with sequence $\left(1^{\left(2^{n}-1\right)} 0\right)$. We wish to perform a move $3: c / d \rightarrow a$. First we do a parallel move to get onto a different branch of the Cayley graph, as in Figure 17. Then we perform $n$ iterations

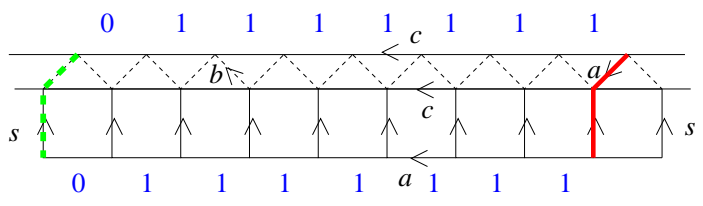

Figure 17. A parallel move to get on a different branch

of move 3 as in Figure 18, Again these segments are concatenated together along the appropriate lines in the Cayley graph. Thus we can find two geodesic words

$$
\begin{gathered}
w=b^{-1} s^{n} a s^{-1} d^{2^{n}} s^{-1} d^{2^{(n-1)}} s^{-1} \ldots s^{-1} d^{4} s^{-1} d^{2} s^{-1} d \\
w^{\prime}=a d s d^{2} s d^{4} s \ldots s d^{2^{(n-1)}} s d^{2^{n}} \operatorname{sas}^{-n},
\end{gathered}
$$

where $w$ is bold and $w^{\prime}$ is a dashed bold path in the figures. It is easily checked, knowing that the sequence on a strip gives all possible geodesics out to that strip, 


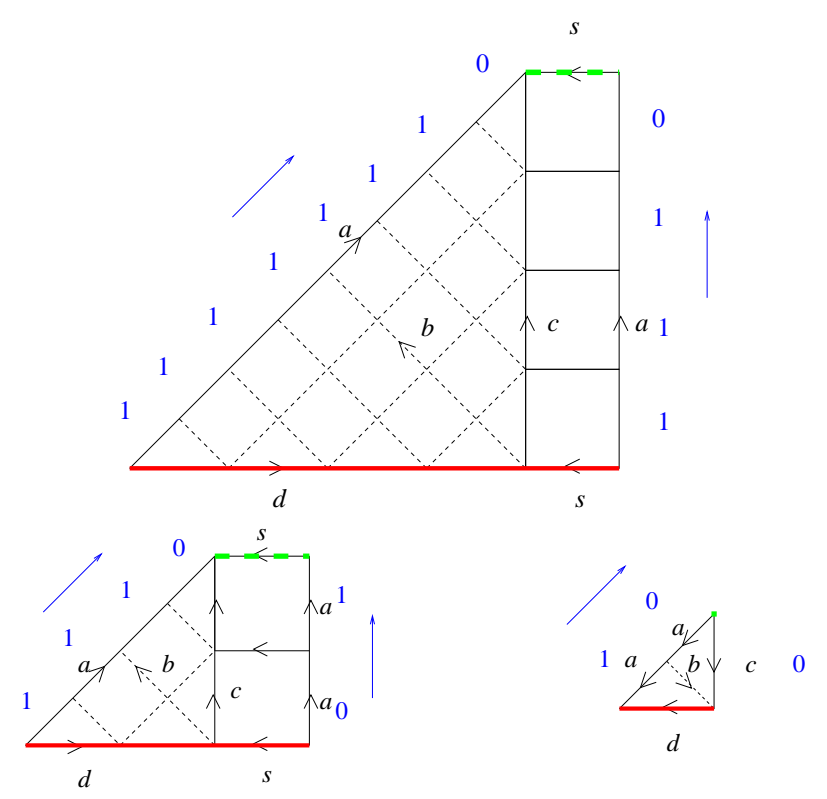

FiguRE 18. "Undoing" the pattern

that $w$ and $w^{\prime}$ are unique geodesics to their endpoints which end an edge apart in the Cayley graph, and fail to $k$-fellow travel for a constant $k$ chosen independently of $n$.

If we glue the pieces together we get a graphic idea of the geodesic structure, as in Figure 19. The parallel move and the strips have been shrunk to lines for simplicity.

Notice that we have shown $\left(G_{1,1}, X\right)$ has no geodesic automatic language, but we have not shown that no geodesic language can be regular, nor that some automatic structure containing at least some non-geodesic representatives does not exist, so the automaticity of this group remains open.

\section{Characterizing patterns}

Theorem 9.1 (Patterns Theorem). All patterns for $\left(G_{1,1}, X\right)$ are of the form

$$
(-1)(0,-1)(0)(1,0)(1) \text {. }
$$

Proof. For any pattern $P$ we say a succession of moves is efficient if the number of moves to get from $T=(-1)(0)(1)$ to a sequence having pattern $P$ is minimal among all successions of moves that give a sequence in $P$. For example, $O^{2}$ is inefficient. We will proceed by induction on the length of efficient successions of moves.

To prove the base step, we know any sequence in $P$ must start at the trivial pattern. Applying moves $0,1,2,3,4$ to $T$ only $T 2$ is non-trivial, so there is only one pattern $T 2$ with an efficient succession of one move.

Now suppose $P$ has an efficient succession of $k$ moves. Suppose $P$ is not of the conjectured form. Then $P$ is non-trivial so an efficient succession of moves for $P$ starts with a 2 . We know that the only move that can give a bad pattern is move 3 , 


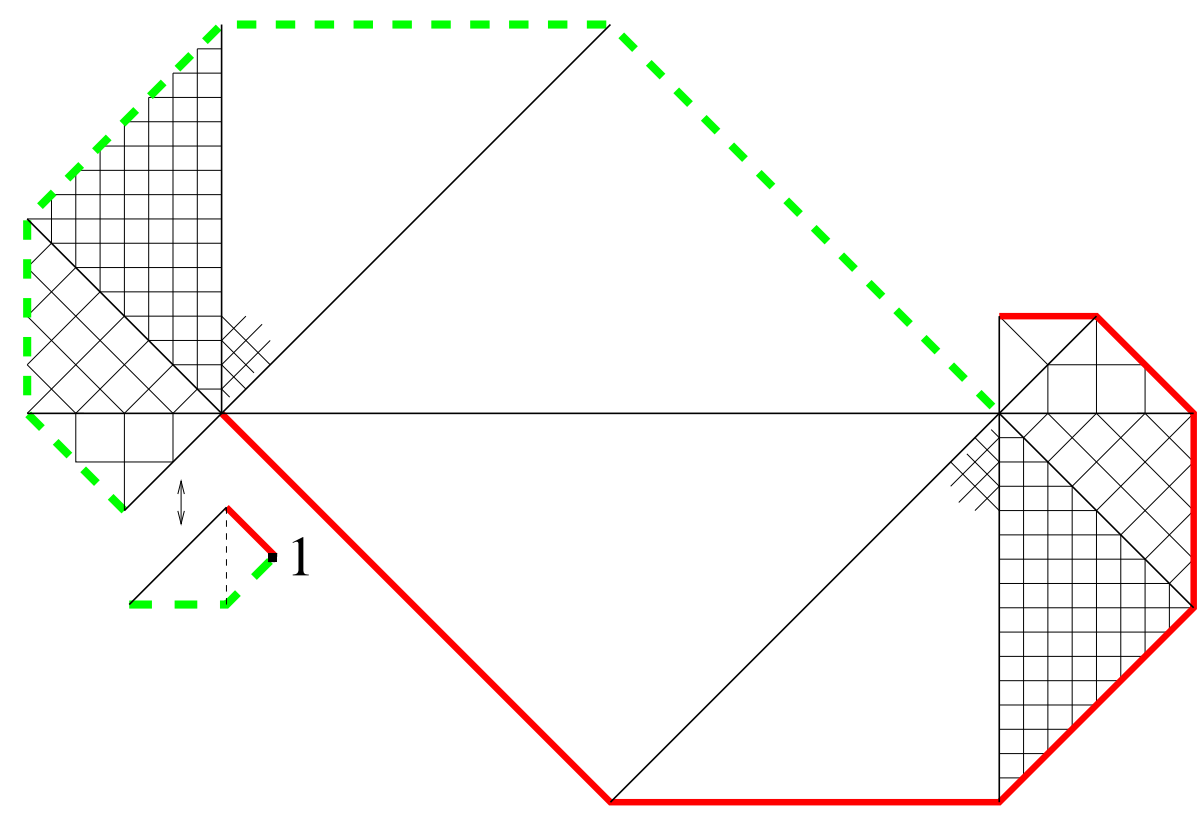

Figure 19. Unique geodesics that don't fellow travel

thus the succession ends with a 3 or there is a succession with less moves that gives a pattern not in the conjectured form. Go to the last 2 in the succession of moves for $P$. Let $K$ be the sequence before the last move 2. So the succession of moves must be $K 2 \ldots 3$. Since it is efficient, we never get more than one 0 concurrently, so the next moves after 2 are either 3 or 03 . Let $K=(-1) w_{1}(0) w_{2} w_{3}(1)$.

$$
K 2=(-1) w_{1}^{\prime}(0-1) w_{2}^{\prime}(0) w_{3}^{\prime}(1)
$$

$K 23$ : Applying move 3 only rewrites the $w_{3}^{\prime}$. Let $w_{3}=x_{1} \ldots x_{n}$. Then $w_{3}^{\prime}=$ $y_{1} \ldots y_{n}$,

$=1 a_{i}$,

$$
y_{i}=\left\{\begin{array}{llll}
1 & 0 & : & x_{i}=0 \\
1 & 1 & : & x_{i}=1
\end{array}\right.
$$

$$
a_{i}=\left\{\begin{array}{ccc}
0 & : & x_{i}=0 \\
1 & : & x_{i}=1
\end{array}\right.
$$

So after the move 3 we get $(-1) w_{3}^{\prime \prime}(1)=(-1) a_{1} a_{2} \ldots a_{n}(1)=(-1) w_{3}(1)$. Thus if $K 23$ is not of conjectured form then neither was $K$, which is contradicts the inductive assumption.

$K 203$ : This time the $w_{1}^{\prime}(0-1) w_{2}^{\prime}$ part is the only part rewritten. Let

$$
\begin{aligned}
& K=(-1) x_{1} \ldots x_{n}(0) x_{n+1} \ldots x_{m} w_{3}(1) \\
& K 2=(-1-1) y_{1} \ldots y_{n}(0-1) y_{n+1} \ldots y_{m}(0) w_{3}^{\prime}(1) \\
& y_{i}=\left\{\begin{array}{rll}
-1-1 & : & x_{i}=-1 \\
0-1 & : & x_{i}=0 \\
0 & : & x_{i}=1
\end{array}\right.
\end{aligned}
$$

Apply move 0:

$$
\ldots(0) p_{m} \ldots p_{n+1}(10) q_{n} \ldots q_{1}
$$




$$
\begin{aligned}
& p_{i}=\left\{\begin{array}{llll}
1 & 0 & : & x_{i}=0 \\
0 & 0 & : & x_{i}=1
\end{array}\right. \\
& =a_{i} 0, \\
& a_{i}=\left\{\begin{array}{lll}
1 & : & x_{i}=0 \\
0 & : & x_{i}=1
\end{array}\right.
\end{aligned}
$$

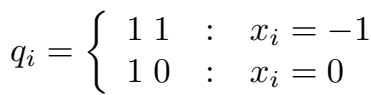

$$
\begin{aligned}
& =1 b_{i}, \\
& b_{i}=\left\{\begin{array}{lll}
1 & : & x_{i}=-1 \\
0 & : & x_{i}=0
\end{array}\right.
\end{aligned}
$$

Now apply move 3: There are two choices for pairing, lets write it out:

$$
\ldots 000 a_{m} 0 \ldots a_{n+1} 010 \ldots 101 b_{n} \ldots 1 b_{1} 111 \ldots
$$

Either choice of pairing gives

$$
\begin{gathered}
(-1) z_{m} \ldots z_{n+1}(0) z_{n} \ldots z_{s}(1), s \leq m \\
z_{i}=\left\{\begin{array}{rll}
-1 & : & a_{i}=0, x_{i}=1 \\
0 & : & a_{i}=1, x_{i}=0 \\
0 & : & b_{i}=0, x_{i}=0 \\
1 & : & b_{i}=1, x_{i}=-1
\end{array}\right. \\
=\left\{\begin{array}{rll}
-1 & : & x_{i}=1 \\
0 & : & x_{i}=0 \\
1 & : & x_{i}=-1
\end{array}\right.
\end{gathered}
$$

Thus $K 203$ is a subsequence of $K 0$. So if $K 203$ is not of conjectured form then neither is $K 0$, but $K 0$ has a shorter efficient succession of moves which contradicts the inductive assumption.

Thus all sequences are of a reasonable form. It would be interesting to characterize the types of sequences that occur. Would they be recognized by some computing machine? That is, to which formal language class might the set of sequences for $\left(G_{1,1}, X\right)$ belong?

\section{Results FOR $\left(G_{W}, Y\right)$}

Recall that the initial patterns for $\left(G_{W}, Y\right)$ are $(-1)(0)(1)$ and $(-1)(10)(1)$. There are 5 types of moves for this example.

$0:$ Reverse orientation of pattern

$1: a \rightarrow a, b \rightarrow b, d \rightarrow d$

$2: d \rightarrow a, d \rightarrow b$

$3: a \rightarrow d, b \rightarrow d$

$4: a \rightarrow b, b \rightarrow a$

Note that move 4 is now non-trivial, in contrast to $\left(G_{1,1}, X\right)$. Following a similar program for this example we can prove a "patterns theorem", that the full language of geodesics is not regular, and that no geodesic automatic structure exists for $\left(G_{W}, Y\right)$. Details are left to the enthusiastic reader, and the same results can be found in [4 for a slightly different (weighted) generating set. 


\section{Almost convexity}

In this section we prove an almost convexity result for multiple HNN extensions which have "well behaved" patterns, that is, which satisfy a condition like that in Theorem 9.1. The proof parallels that given by the author in [5] for different hypotheses.

Definition 11.1 (Almost convex). $(G, X)$ is almost convex if there is a constant $C$ such that every pair of points lying distance at most 2 apart and within distance $N$ of the identity in $\Gamma(G, X)$ are connected by a path of length at most $C$ which lies within distance $N$ of the identity.

See [3] for properties of almost convex groups.

Definition 11.2 (Falsification by fellow traveler property). $(G, X)$ enjoys the falsification by fellow traveler property if $\exists k$ such that every non-geodesic word $w \in X^{*}$ is $k$-fellow traveled by a shorter word with the same start and end points.

If $(G, X)$ has the falsification by fellow traveler property then the language of all geodesics on $X$ is regular [10]. It follows that $\left(G_{1,1}, X\right)$ does not enjoy this property. If $(G, X)$ has the falsification by fellow traveler property then it is almost convex, with constant $C=3 k$. Both properties depend on the choice of generating set.

Definition 11.3 (Well behaved). Let $(G, X)$ be a multiple HNN extension as in Definition 3.1 with a strip equidistant presentation. We say $(G, X)$ has well behaved patterns if each pattern is of the form $(-1,0)(0)(1,0)$.

Theorem 11.4. Let $(G, X)$ be a multiple $H N N$ extension of $(A, Z)$ as in Definition 3.1 with a strip equidistant presentation, such that associated subgroups are geodesic, $(A, Z)$ enjoys the falsification by fellow traveler property and $(G, X)$ has well behaved patterns. Then $(G, X)$ is almost convex.

Remark: In [5] the well behaved patterns hypothesis is absent, and instead we require that associated subgroups are totally geodesic. Wise's example satisfies either set of hypotheses, while $\left(G_{1,1}, X\right)$ has well behaved patterns but fails to have totally geodesic associated subgroups, as noted in Examples 6 6 7 above. Neumann and Shapiro prove that an abelian group with any finite generating set has the falsification by fellow traveler property [10, so it follows that both examples are almost convex.

Proof. Let $S(N)$ denote the metric sphere of radius $N$ and $B(N)$ the metric ball of radius $N$ in $\Gamma(G, X)$. Let $g, g^{\prime} \in S(N)$ with $d\left(g, g^{\prime}\right) \leq 2$ realized by a path $\gamma$. Let $w, u$ be geodesic words for $g, g^{\prime}$ respectively. Since the presentation is strip equidistant, $w, u$ are stable letter reduced. Let $k$ be the falsification by fellow traveler property constant for $(A, Z)$ and assume that $k$ is an even integer greater than $\max \left\{\left|u_{i_{j}}\right|: u_{i_{j}}\right.$ is a generator of $\left.U_{i} \forall i\right\}$.

Case 1. $w, u, \gamma$ have no stable letters. The word $w \gamma$ is not geodesic in $(A, X)$, so applying the falsification by fellow traveler property we can find a shorter word $q$ which ends at $g^{\prime}$ and $k$-fellow travels it. If $q$ is not geodesic then we can find a shorter word $y$ which ends at $g^{\prime}$ and $k$-fellow travels $q$. Moreover $y$ is a geodesic so has length $N$. The path that retraces $w$ back to $w\left(N-\frac{k}{2}\right)$, then across to $q\left(N-\frac{k}{2}\right)$ by a path of length at most $k$, then across to $y\left(N-\frac{k}{2}\right)$ by a path of length at most $k$, then along $y$ to $g^{\prime}$, stays within $B(N)$ and has length at most $3 k$. 
Case 2. $\gamma$ involves a stable letter. Then $\gamma=s, s x, x s$ for $s \in\left\{s_{i}^{ \pm 1}\right\}_{i=1}^{n}$ and $x$ any generator or inverse of a generator except $s^{-1}$. By Britton's Lemma $w \gamma u^{-1}$ contains a pinch so either $w$ or $u$ has an $s^{-1}$. Without loss of generality assume $w=w_{1} s^{-1} w_{2}$.

Case 2a. If $\gamma=s, s x$ then we have Figure 20(a). $w_{2}$ is geodesic and evaluates to

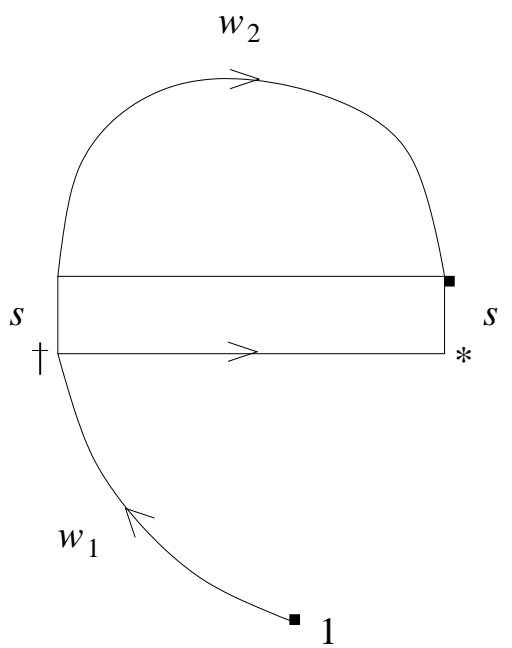

(a) Case 2a

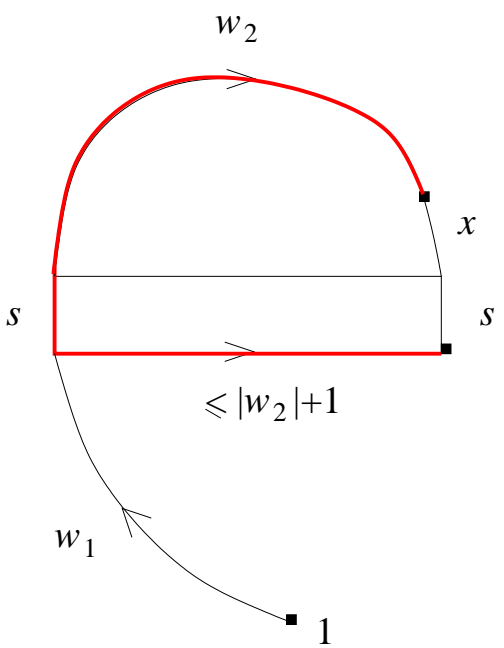

(b) Case $2 b$

FiguRE 20.

an element of an associated subgroup $U_{i}$ or $V_{i}$. of $U_{i}$. The point $\overline{w_{1}}=\dagger$ lies in $B\left(N-\left|w_{2}\right|-1\right)$ and since associated subgroup words are geodesic, the point $\overline{w s}=*$ lies in $B(N-1)$ so $\gamma=s x$ lies in $B(N)$ and $\gamma=s$ gives a contradiction.

If $\gamma=x s$ then $w_{2} x$ evaluates to a subgroup element. If $x$ is a stable letter other than $s^{-1}$ then we have the previous case. So $w_{2} x \in Z^{*}$, and there are two further subcases to consider, depending on $\left|w_{2}\right|$.

Case 2b. If $\left|w_{2}\right| \leq \frac{k}{2}$ then the path that retraces $w$ to $\overline{w_{1}}$ then travels along the bottom of the strip to $g^{\prime}$, shown in bold in Figure 20(b), lies inside $B(N)$ and has length at most $k+2$.

Case 2c. If $\left|w_{2}\right|>\frac{k}{2}$ then we can argue as follows. Let $v$ be the geodesic word in the associated subgroup for $w_{2} x$ and let $z$ be the last generator $u_{i}$ (or $v_{i}$ ) of $v$, and let $z_{2}$ be the last letter in $Z$ of $z$. That is, $v=v^{\prime} z=v^{\prime} z_{1} z_{2}$. The path $w_{2} x z_{2}^{-1}$ is not geodesic in $(A, Z)$, so applying the falsification by fellow traveler property at most twice we find a path $y$ of length at most $\left|w_{2}\right|$ which $2 k$-fellow travels it.(That is, $w_{2} x z_{2}^{-1}$ and $y k$-fellow travel an intermediate path $q$. See Figure 21. Then $y$ lies in $B(N)$ since it starts at the point $\overline{w_{1} s} \in B\left(N-\left|w_{2}\right|\right)$. We can now find a path connecting $g$ and $g^{\prime}$ inside $B(N)$ as follows: Retrace $w$ back to $w\left(N-\frac{k}{2}\right)$, then across to $w_{1} s y\left(N-\frac{k}{2}\right)$, along $y$ to its end, then around the last relator of the strip, shown in bold in Figure 21, lies in $B(N)$ and has length at most $3 k+2 \max \left\{\left|u_{i_{j}}\right|: u_{i_{j}}\right.$ is a generator of $\left.U_{i} \forall i\right\} \leq 5 k$. 

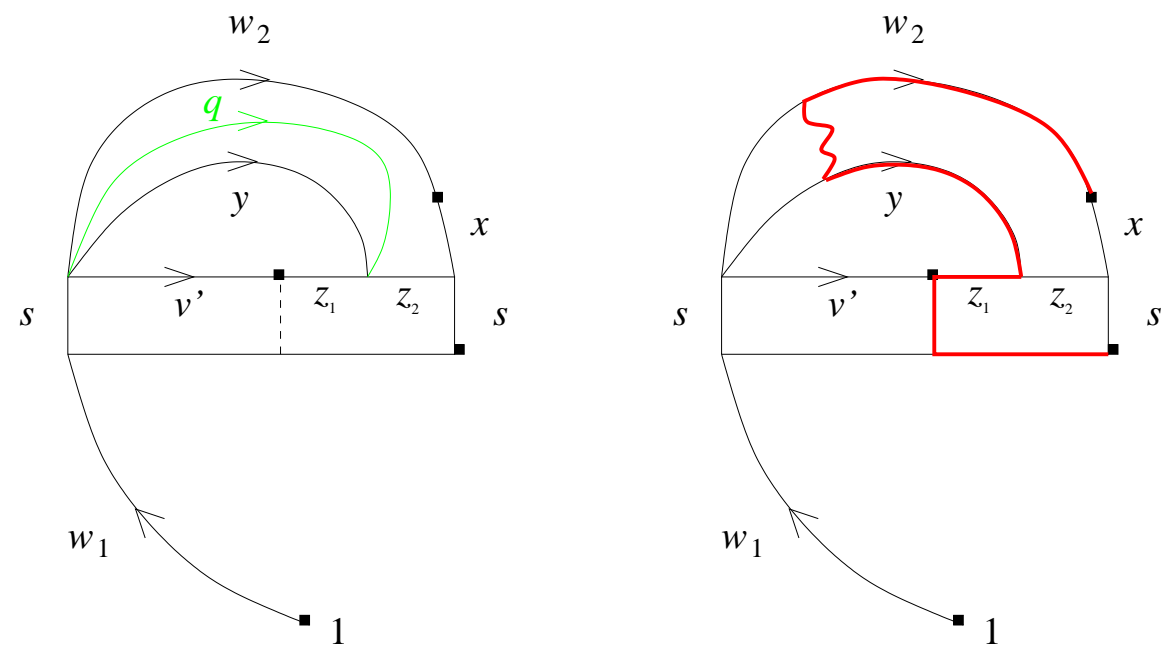

Figure 21. Case 2c

Case 3. $\gamma$ has no stable letters and $w$ has a stable letter. Then $w, u$ have parallel stable letter structure. Let $s \in\left\{s_{i}^{ \pm 1}\right\}$ be the last stable letter of $w$, so $w=$ $w_{1} s w_{2}, u=u_{1} s u_{2}$, and $w_{2} \gamma u_{2}^{-1} \in Z^{*}$ and evaluates to an associated subgroup element.

Case 3a. If $\left|w_{2}\right|,\left|u_{2}\right| \leq \frac{k}{2}$ then the path that retraces $w$ to the last strip, then runs across the bottom of the strip, then back along $s u_{2}$, shown in bold in Figure 22. lies within $B(N)$ and has length at most $4 k+2$.

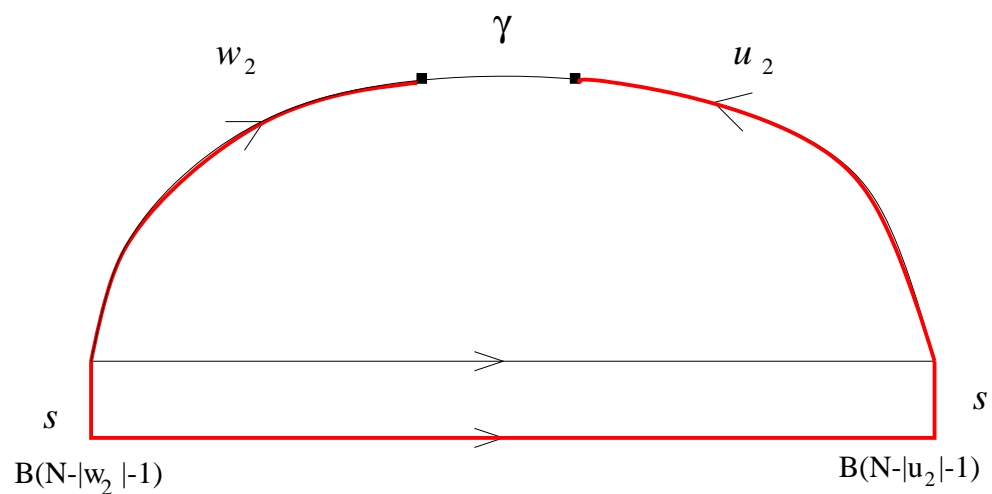

Figure 22. Case 3a: $\left|w_{2}\right|,\left|u_{2}\right| \leq \frac{k}{2}$.

Otherwise at least one of $\left|w_{2}\right|>\frac{k}{2}$ or $\left|u_{2}\right|>\frac{k}{2}$. Without loss of generality assume $\left|w_{2}\right| \geq\left|u_{2}\right|$, so $\left|w_{2}\right|>\frac{k}{2}$.

Case $3 b$. If $w_{2} \gamma u_{2}^{-1}$ is geodesic then it may not necessarily be a word in the associated subgroup, that is, it may not run along the top of the last strip. Let $v$ be 
a geodesic for it in the associated subgroup. Let $z$ be the last $|\gamma|$ letters of $v$, so $v=v^{\prime} z$, and $\left|v^{\prime}\right|=\left|w_{2}\right|+\left|u_{2}\right|$. The word $w_{2} \gamma u_{2}^{-1} z^{-1}$ has length $\left|w_{2}\right|+\left|u_{2}\right|+|\gamma|+|z|$ so is not geodesic, so applying the falsification by fellow traveler property in $(A, Z)$ at most four times we can find a geodesic path $y$ for $w_{2} \gamma u_{2}^{-1} z^{-1}$ which $4 k$-fellow travels it (with three intermediate paths).

Now since patterns are well behaved, the point $\overline{w_{1} s v^{\prime}}$ labeled by $*$ in Figure 23 must lie in $B\left(N-\left|u_{2}\right|\right)$, thus the path $y$ lies in $B(N)$. The path that retraces $w$

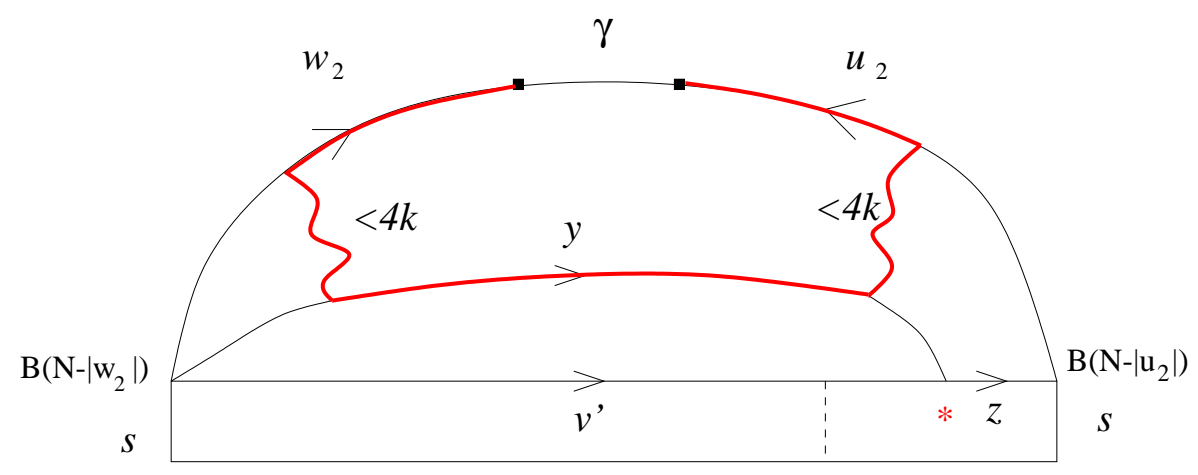

Figure 23. Case $3 \mathrm{~b}:\left|w_{2}\right|>\frac{k}{2}, w_{2} \gamma u_{2}$ geodesic.

to $w\left(N-\frac{k}{2}\right)$, then across to $y\left(N-\frac{k}{2}\right)$, then along $y$ to $y\left(N+\frac{k}{2}+|\gamma|\right)$, then across to $u\left(N-\frac{k}{2}\right)$ and back to $g^{\prime}$ along $u$, shown in bold in Figure 23, lies within $B(N)$ and has length at most $10 k+2$. If $\left|u_{2}\right|<\frac{k}{2}$ then the path would follow $y$ until its end, go around the last relator of the strip (which contains $z$ ) and along $u_{2}$.

Case 3c. If $w_{2} \gamma u_{2}^{-1}$ is not geodesic then by the falsification by fellow traveler property in $(A, Z)$ there is a path $y$ which $k$-fellow travels it. If $y$ is geodesic, we can repeat the previous argument to obtain a path of length at most $10 k+2$ inside $B(N)$. The details are omitted.

Case $3 d$. If $y$ is also not geodesic then applying the falsification by fellow traveler property once more we get a path $p$ of length at most $\left|w_{2}\right|+\left|u_{2}\right|+|\gamma|-2 \leq\left|w_{2}\right|+\left|u_{2}\right|$, which runs from $\overline{w_{1} s}$ to $\overline{u_{1} s}$ so lies in $B(N)$. Then the path that retraces $w$ to $w\left(N-\frac{k}{2}\right)$, then across to $y\left(N-\frac{k}{2}\right)$, then across to $p\left(N-\frac{k}{2}\right)$, along $p$ to $p\left(N+\frac{k}{2}+|\gamma|\right)$, over to $u\left(N-\frac{k}{2}\right)$ and back to $g^{\prime}$, shown in bold in Figure 24, lies within $B(N)$ and has length at most $6 k+2$. If $\left|u_{2}\right|<\frac{k}{2}$ then the path would follow $p$ until its end, then run along $u_{2}$.

In each case the maximum length of a path needed to connect $g$ to $g^{\prime}$ inside $B(N)$ is $10 k+2$, and the result follows.

\section{REFERENCES}

[1] Noel Brady and Martin Bridson. On the absense of biautomaticity for graphs of abelian groups. Unpublished.

[2] Martin R. Bridson and André Haefliger. Metric spaces of non-positive curvature. SpringerVerlag, Berlin, 1999.

[3] James W. Cannon. Almost convex groups. Geom. Dedicata, 22(2):197-210, 1987. 


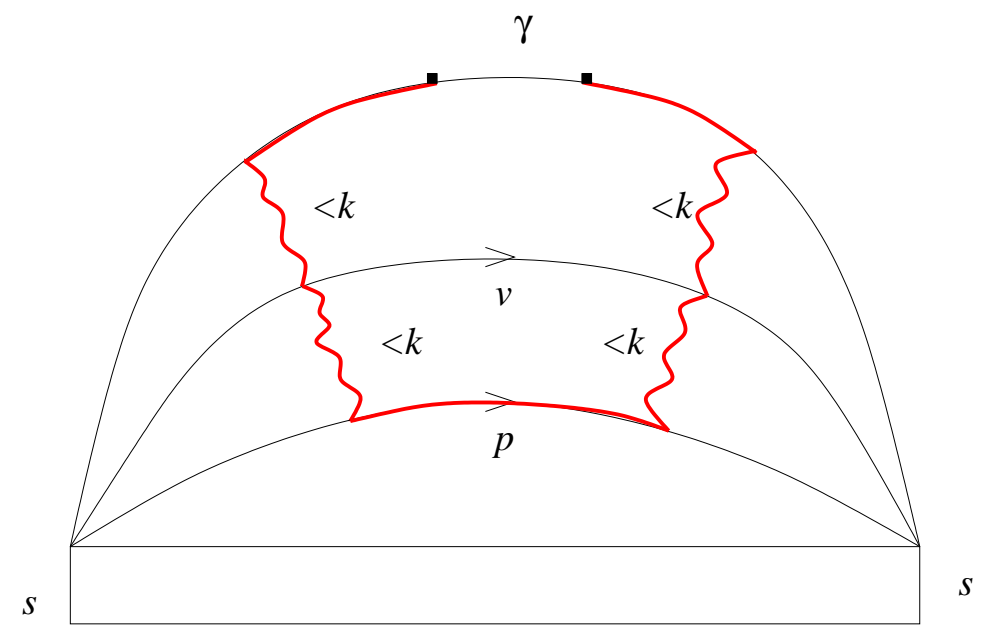

Figure 24. Case 3d: $\left|w_{2}\right|>\frac{k}{2}, w_{2} \gamma u_{2}, y$ not geodesic.

[4] Murray Elder. Automaticity, almost convexity and falsification by fellow traveler properties of some finitely generated groups. PhD Dissertation, University of Melbourne, 2000.

[5] Murray J. Elder. A non-Hopfian almost convex group. J. Algebra, 271(1):11-21, 2004.

[6] David B. A. Epstein, James W. Cannon, Derek F. Holt, Silvio V. F. Levy, Michael S. Paterson, and William P. Thurston. Word processing in groups. Jones and Bartlett Publishers, Boston, MA, 1992.

[7] S. M. Gersten. The automorphism group of a free group is not a cat(0) group. Proc. Amer. Math. Soc., 121(4):999-1002, 1994.

[8] John E. Hopcroft and Jeffrey D. Ullman. Introduction to automata theory, languages, and computation. Addison-Wesley Publishing Co., Reading, Mass., 1979. Addison-Wesley Series in Computer Science.

[9] Roger C. Lyndon and Paul E. Schupp. Combinatorial group theory. Springer-Verlag, Berlin, 1977. Ergebnisse der Mathematik und ihrer Grenzgebiete, Band 89.

[10] Walter D. Neumann and Michael Shapiro. Automatic structures, rational growth, and geometrically finite hyperbolic groups. Invent. Math., 120(2):259-287, 1995.

[11] Daniel T. Wise. A non-Hopfian automatic group. J. Algebra, 180(3):845-847, 1996.

(As at Dec 2006) Dept of Mathematical Sciences, Stevens Institute of Technolgy, HoBOKEn NJ 07030 USA

E-mail address: melder@stevens.edu 